Literarisches Leben im antiken Rom 


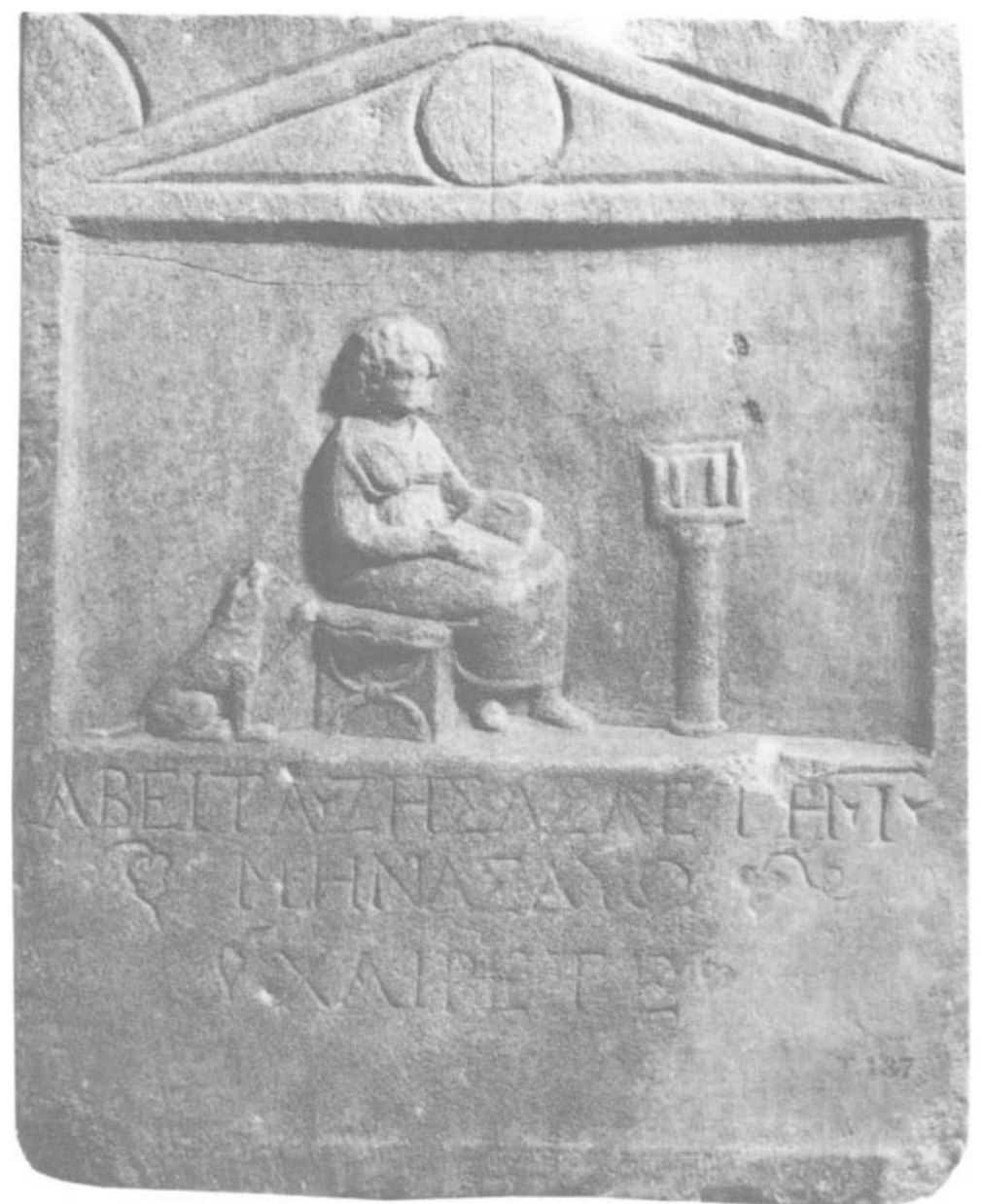


Elaine Fantham

\section{Literarisches Leben im antiken Rom}

Sozialgeschichte der römischen Literatur von Cicero bis Apuleius

Aus dem Englischen von Theodor Heinze

Verlag J. B. Metzler Stuttgart $\cdot$ Weimar 
Titel der amerikanischen Originalausgabe:

Elaine Fantham: Roman Literary Culture. From Cicero to Apuleius

(C) 1996 The Johns Hopkins University Press

Frontispiz: Grabrelief der Avita, vermutlich aus dem frühen 3. Jh. n. Chr., gefunden in Rom. Das Relief ist aus kleinasiatischem Marmor gefertigt und trägt eine Inschrift in griechischer Sprache. Hier zeigt sich die kulturelle Dominanz der griechischen Sprache und Kunst.

British Museum Sculpture 649. (C) The British Museum.

Die Deutsche Bibliothek - CIP-Einheitsaufnahme

\section{Fantham, Elaine:}

Literarisches Leben im antiken Rom : Sozialgeschichte der römischen Literatur von Cicero bis Apuleius / Elaine Fantham.

Aus dem Engl. von Theodor Heinze.

- Stuttgart ; Weimar : Metzler, 1998

ISBN 978-3-476-01592-1

ISBN 978-3-476-01592-1

ISBN 978-3-476-03734-3 (eBook)

DOI 10.1007/978-3-476-03734-3

Dieses Werk einschließlich aller seiner Teile ist urheberrechtlich geschützt. Jede Verwertung außerhalb der engen Grenzen des Urheberrechtsgesetzes ist ohne Zustimmung des Verlages unzulässig und strafbar. Das gilt insbesondere für Vervielfältigungen, Übersetzungen, Mikroverfilmungen und die Einspeicherung und Verarbeitung in elektronischen Systemen.

(C) 1998 Springer-Verlag GmbH Deutschland Ursprünglich erschienen bei J. B. Metzlersche Verlagsbuchhandlung und Carl Ernst Poeschel Verlag GmbH in Stuttgart 1998 


\section{Inhalt}

Vorwort und Danksagungen $\ldots \ldots \ldots \ldots \ldots \ldots \ldots$. . . . . . . . . .

EIN LE IT U N G: Auf dem Weg zu einer Sozialgeschichte

der lateinischen Literatur . . . . . . . . . . . . . . . . . . 1

Autor, Publikum und Medium . . . . . . . . . . . . . . . 2

Ennius und Cato, zwei frühe Schriftsteller . . . . . . . . . . . 10

Neue Gattungen der Literatur von Lucilius bis Apuleius . . . . . . . . . 11

Bemerkungen zur Gattungstheorie . . . . . . . . . . . . . . 16

KA PITEL 1: Rom am Ende der Republik . . . . . . . . . . . . . 18

Unterricht und Bildung in Rom - und was dabei herauskam . . . . . . . 21

Literatur und Nationalismus . . . . . . . . . . . . . . . . . . . . . . . . . . . . . . . . .

Literatur und ihre Liebhaber . . . . . . . . . . . . . . . . . 37

Studien zur Literatur und das Hobby der Literaturgeschichte . . . . . . . . 38

Literatur und Gelehrsamkeit: Caesar undVarro . . . . . . . . . . . . 43

KA PITEL 2: Die Heraufkunft des Prinzipats: Literarisches Leben in raugusteischer Zeit . . . . . . . . . . . . . . . 50

Zwei Überlebende: Die `Neuen Dichterı Gallus und Vergil . . . . . . . . . 51

Das römische Gedichtbuch, eine neue literarische Form . . . . . . . . 57

Privates und öffentliches Mäzenatentum . . . . . . . . . . . . . . . 61

Der Kaiser als Thema und Mäzen . . . . . . . . . . . . . . . . 68

Der beste aller Patrone und sein Großer Freund ～. . . . . . . . . . 70

Dichterlesungen und Leserschaft . . . . . . . . . . . . . . . 77

Mündliche und schriftliche Prosa in der augusteischen Gesellschaft:

Rhetorik als Training und Show . . . . . . . . . . . . . . . . . . . . 83

Die ersten echten Geschichtswerke . . . . . . . . . . . . . . 87

KA PITEL 3: Unaugusteische Aktivitäten . . . . . . . . . . 95

Die Literatur der Jugend $\ldots \ldots \ldots$

Liebe und Elegie . . . . . . . . . . . . . . . . . . . . . . . . . 99

Ovid als Sündenbock und der Kummer des Augustus . . . . . . . . . . . 104

Unschuld und Macht des Buches . . . . . . . . . . . . . . . . . . 110 


\section{Inbalt}

KA PITEL 4: Eine Generation, die sich nicht entfalten kann:

Unterdrückung und Überleben . . . . . . . . . . . . . . . . . . . . 118

Erlaubte Literatur: Prosa . . . . . . . . . . . . . . . . . . . . . . 120

Moralische Traktate und Briefe . . . . . . . . . . . . . . 126

Didaktische und beschreibende Dichtung . . . . . . . . . . . . 128

Forscher, gentleman und Angeber . . . . . . . . . . . . . . . . . 131

Vorlieben und Vorurteile der kaiserlichen Nachfolger des Augustus . . . . . 131

Die Auseinanderentwicklung von Theater und Drama . . . . . . . . . . . 136

KA PITEL 5: Zwischen Nero und Domitian: Die Herausforderung an die Dichtung . . . . . . . . . . . . . . . . . 143

Die neronische Renaissance . . . . . . . . . . . . . . . . 143

Dichtung und Parodie in einer neuen Umgebung . . . . . . . . . . . 153

Wechselfälle der epischen Muse . . . . . . . . . . . . . . . . 157

Professionelle Dichter in der Zeit des Domitian . . . . . . . . . . . . . 162

KA PITEL 6: Literatur und die herrschenden Schichten:

Von Vespasians Regierungsantritt bis zu Trajans Tod . . . . . . . . . . 173

Autoren aus dem Ritter- und Senatorenstand: Eine sich wandelnde

Oberschicht . . . . . . . . . . . . . . . . . . . . . . . . . 173

Alternativen einer literarischen Karriere: Ruhm oder Überleben . . . . . 180

Plinius' Briefe und seine literarische Welt . . . . . . . . . . . . . . . 189

Das Leben des Senators und des Redners in der Öffentlichkeit . . . . . . . 192

Die Welt des Auditoriums . . . . . . . . . . . . . . . . . . . . . . . . . 199

KA PITEL 7: Literarische Kultur im Niedergang:

Die antoninischen Jahre . . . . . . . . . . . . . . . . . 210

Hadrian, der Philhellene . . . . . . . . . . . . . . . . . . 210

Die reisenden Sophisten . . . . . . . . . . . . . . . 217

Die Provinzen und die lateinische Kultur . . . . . . . . . . . . 223

Marcus Aurelius und seine Lehrer . . . . . . . . . . . . . . . . 227

Aulus Gellius, der ewige Student in Rom und Griechenland . . . . . . . 234

Apuleius, der vollendete Wortkünstler . . . . . . . . . . . . . . . . 240

Anmerkungen . . . . . . . . . . . . . . . . . . 252

Abkürzungsverzeichnis . . . . . . . . . . . . . . . . . . . . . . 294

Bibliographie . . . . . . . . . . . . . . . . . . . . 296

Register der Namen und Begriffe . . . . . . . . . . . . . . . . 303 


\section{Vorwort und Danksagungen}

Lassen Sie mich mit einer Einschränkung beginnen. Dieses Buch will keine Literaturgeschichte sein. Eine solche erforderte die seltene Verbindung einer klaren Vorstellung vom Ganzen mit dem eindringenden Verständnis verschiedenster Gattungstraditionen und schöpferischer Persönlichkeiten. Selbst diejenigen, die solche literaturkritischen Talente in höchstem Maße besitzen, haben ihre Vorbehalte angesichts der Schwierigkeit eines so ehrgeizigen Projektes angemeldet. Die divergierenden Ziele der Erarbeitung einer historischen Entwicklung einerseits und der Bereitstellung eines literaturkritischen Instrumentariums und der Würdigung andererseits geraten oft miteinander in Konflikt, oft werden Autoren ungleich behandelt. Glücklicherweise steht englischsprachigen Studenten und Lehrern jetzt ein klarer und kohärent strukturierter analytischer Überblick über die römische Literatur aus der Feder eines hervorragenden Gelehrten zur Verfügung. Die Veröffentlichung einer überarbeiteten und erweiterten englischsprachigen Version von Gian Biagio Contes Buch Letteratura latina: Manuale storico dalle origini alla fine dellimpero romano (engl. unter dem Titel: Latin Literature: $A$ History) ist für mich ein gewichtiger Grund, eine von ihm so glänzend bewältigte Aufgabe nicht noch einmal angehen zu wollen.

Stattdessen habe ich versucht, ein Begleitbuch vorzulegen, das Schülern und Studenten Nutzen bringen soll, die mit den Hauptautoren der lateinischen Literatur und den groben Zügen der römischen Geschichte bereits vertraut sind. Etwas ehrgeiziger ist es vielleicht, wenn ich Studenten anderer Literaturepochen und einem größeren Publikum mit profundem Interessse an lateinischer Literatur und ihrem sozialen Kontext einige neue Perspektiven aufzuzeigen hoffe. I. A. Richards und die Pioniere des Practical Criticism würden einem solchen Projekt gewiß nicht beipflichten. Ihr Hauptbeitrag zur Literaturkritik lag jedoch in der Lyrikinterpretation und der Interpretation von Dichtung der letzten zwei Jahrhunderte. Klassische Philologen haben immer schon ein close reading praktiziert, ein textnahes Interpretationsverfahren, das sich nicht sehr von dem des Practical Criticism unterscheidet, doch haben sie zumeist anerkannt, daß es beim Sprung über die Jahrtausende hinweg unabdingbar ist, den Kontext von Literatur zu berücksichtigen. Im Rahmen der gegenwärtigen Erneuerung eines Interesses am historischen Kontext (dem New Historicism) erscheint eine Sozialgeschichte der römischen Literatur wohl weniger befremdlich. Selbst wenn wir uns mit den großen Texten in Dichtung und Prosa 
hinreichend vertraut fühlen, um uns unser eigenes ästhetisches Urteil zu bilden, kann die Kenntnis des sozialen und kulturellen Kontextes unsere Wertschätzung des einzelnen Werks modifizieren oder schärfen. Deshalb ist jene Welt Gegenstand meiner Darstellung, in der diese Literatur ihre Entwicklung von der Reife um $50 \mathrm{v}$. Chr. bis zum Niedergang in der Generation nach $150 \mathrm{n}$. Chr. durchlief.

Für ein solches Buch hätte ein thematischer Aufbau mit einzelnen Kapiteln zur Zweisprachigkeit, zur mündlichen Kommunikation oder zum Mäzenatentum in diesen zwei Jahrhunderten nahegelegen. Stattdessen werde ich chronologisch vorgehen, von einer Generation zur nächsten, und einzelnen kanonischen Autoren in aufeinanderfolgenden Kapiteln besondere Aufmerksamkeit schenken. Wahtscheinlich werden viele Studenten zu diesem Buch greifen, um sich bei der Lektüre der WerkeVergils, Ovids, Senecas, Plinius' des Jüngeren oder des Apuleius Hintergrundwissen zu erarbeiten, so daß ein solcher Aufbau sie schneller ans Ziel führen wird. Doch gibt es einen gewichtigeren Grund für dieses Vorgehen: Die römische Gesellschaft war in beinahe jeder Hinsicht einem raschen Wandel unterworfen. So ist die Wirklichkeit von Ovids Welt zum Beispiel durch das Zusammentreffen einer Reihe von Veränderungen bedingt: dem Entstehen öffentlicher Bibliotheken, der Absorption der Rhetorik durch die Deklamationsschulen, der Ausweitung der Leserschaft subjektiver Dichtung (im Hinblick auf Alter, Geschlecht und Geographie) und dem Verschwinden des Mäzenatentums außerhalb des Kaiserhauses.

Was verstehen wir unter römischer Literatur? Im zweiten, der lateinischen Literatur gewidmeten Band der Cambridge History of Classical Literature wurde offenkundig die Ansicht vertreten, daß Literatur im wesentlichen kreative oder imaginative Schriftstellerei sei, ob es sich um Dichtung oder (was in Rom seltener der Fall ist) um Prosa handele; der Dichtung und dem Roman wurde mehr Raum gegeben als der Geschichtsschreibung (obwohl die römische Geschichtsschreibung der Fiktion oft näherkommt als der objektiven Darstellung) und geographischen, juristischen, naturwissenschaftlichen oder literaturgeschichtlichen Werken. Auch den griechischen Schriftstellern und Lehrern in Rom wurde wenig Platz eingeräumt, obwohl diese Männer einen so außergewöhnlichen Einfluß auf Autoren und Publikum hatten. Doch hat die verschiedene Elizabeth Rawson diesen und viele andere Aspekte in ihrem Buch Intellectual Life in the Later Roman Republic schon glänzend und erschöpfend dargestellt.

Heutzutage sind Buchhandlungen in Rußland, Italien oder Holland voll von Übersetzungen fiktionaler Literatur aus dem Amerikanischen und von politischen oder philosophischen Titeln aus Frankreich oder Deutschland; derlei wird von den Leuten gelesen, genauso wie junge Amerikaner jetzt fiktionale Literatur lesen, die aus dem argentinischen oder mexikanischen Spanisch übersetzt wurde, und Studenten der Theaterwissenschaft sich überall mit Ibsen und Strindberg beschäftigen. Es wäre unrealistisch, wenn man über amerikanische oder russische Schriftsteller unserer Zeit ein Urteil abgeben würde, als hätten sie sich mit diesen Vorlagen aus dem 
Ausland nie auseinandergesetzt, als wären sie nie dem Einfluß dieser formalen und inhaltlichen Innovationen ausgesetzt gewesen. Es hat in Rom vielleicht wenig wörtliche Übersetzungen griechischer Werke gegeben, doch wurde die literarische Erfahrung gebildeter Römer in dem Maße, wie sie Griechisch beherrschten, von griechischen Texten, griechischen Lehrern und griechischen Vortragenden geprägt, und das Griechische scheint zumindest für einen Teil des 2. Jahrhunderts n. Chr. seine Vorherrschaft über das Lateinische erneuert zu haben. Daher werden die Frage der Zweisprachigkeit und die Rolle zeitgenössischer Griechen in der literarischen Welt der Römer in dieser Sozialgeschichte` immer wieder thematisiert werden.

Auch in einer weiteren Hinsicht habe ich versucht umfassend zu sein: Römische Literatur meint zunächst die Literatur, die man in Rom bzw. Italien, dem nächsten konzentrischen Ring um Roms Bürgerschaft und Kultur, las und schrieb. Doch wurde römische Literatur zu Beginn und auch später von Männern geschrieben, die nicht aus dem überkommenen Kulturbereich stammten: von Livius Andronicus und Ennius, die beide Griechisch sprachen, von Plautus aus Umbrien, von Catull und Vergil aus Gallia Cisalpina nördlich des eigentlichen Italiens, und, als Rom sich ausdehnte, von Seneca, Martial und Quintilian aus Spanien sowie Fronto und Apuleius aus Nordafrika. Niemand zweifelt daran, daß Seneca ein römischer Schriftsteller war, denn er machte in Rom Karriere und starb auch dort. Bei Apuleius scheint das schon anders zu sein: Er absolvierte einen Teil seines Bildungsweges in Rom und kehrte später auch dorthin zurück, doch hinterließ seine Ausbildung in Athen die deutlicheren Spuren in seinem Werk. Rom war nicht mehr das Zentrum literarischer Produktivität in lateinischer Sprache, so daß eine Untersuchung der literarischen Kultur der Römer in Apuleius' Zeit zur Untersuchung der literarischen Kultur lateinischer Sprache wird, wo sie am höchsten entwickelt war in Nordafrika und seiner gesellschaftlichen wie geistigen Hauptstadt Karthago. Doch angesichts des beginnenden Schweigens der paganen lateinischen Literatur, das nach Apuleius' Tod zwei Jahrhunderte lang andauern sollte, und weil die wachsende Dominanz des Christentums zum Thema nordafrikanischer Eloquenz wird, scheint es legitim, mit der kulturellen Welt des Apuleius abzuschließen. Apuleius' Welt, die römischer als Hadrians Gesellschaft und vitaler als Iuvenals bitter-satirisches Bild der Hauptstadt ist, stellt einen prächtigen Abschluß dar - der auf eine noch zu wenig erforschte Gesellschaft hinweist.

Mein Ziel bestand also darin, die Veränderungen in der Gesellschaft zu beleuchten, in der römische Schriftsteller schrieben und ihr Publikum fanden, die kulturellen Vorgaben und Erwartungen nachzuzeichnen, mit denen das Publikum bzw. die Leser Autoren oder Texte erstmals rezipierten, und dort, wo es angebracht ist, Aufstieg und Niedergang verschiedener Gattungen durch gesellschaftlichen und politischen Wandel zu erklären. Bei der Weite des Themas und dem Reichtum an interessanten neuen Veröffentlichungen zu einzelnen Autoren und Epochen hatte 
ich eine persönliche Auswahl zu treffen, was besonders in den beiden Kapiteln nicht zu übersehen ist, die dem komplexen Zusammenhang von privater und gesellschaftlicher Kultur im augusteischen Zeitalter gewidmet sind; doch kann der Leser zu anderen Darstellungen aus jüngster Zeit greifen, wenn er eingehendere Informationen zu dieser maßgebenden Epoche sucht.

Viele Kollegen und Schüler haben mich bei der Entstehung dieses Buches unterstützt. Ich verdanke zu vielen Freunden, lebenden wie verschiedenen, zu viel, als daß ich allen gerecht werden könnte; meinen besonderen Dank möchte ich dennoch Ted Champlin für seine sorgfältige Lektüre der Kapitel 6 und 7, Maud Gleason für die kritische Durchsicht meiner kurzen Darstellung der Zweiten Sophistik, und vor allem Gian Biagio Conte aussprechen, letzterem dafür, daß er mir die überarbeiteten Abschnitte zu vielen Autoren in seiner Letteratura latina noch vor der Veröffentlichung zugänglich machte, und für die Zeit, die er erst kürzlich aufwandte, um seine Gedanken zur literarischen Kultur der neronischen Zeit, wie Petron sie sah (und prendeva in giro), mit mir zu diskutieren - doch vor allem für seine moralische Unterstützung in schwierigen Zeiten.

Ich habe auch vielen Institutionen zu danken: meiner eigenen Universität, Princeton, für Beurlaubungen in den Jahren 1991 und 1995 und Unterstützung aus dem Humanities Research Fund, dem Pembroke College und der Faculty of Classics, Cambridge, für ihre Gastfreundschaft und wunderbare Arbeitsumgebung, dem Dipartimento di Studi Classici der Universität Pisa für den herzlichen Empfang eines inoffiziellen Gastes und der American Academy für das ehrenvolle Privileg, auf dem Gipfel des Monteverde wohnen, ihrer Gemeinschaft angehören und die Ruhe ihrer Bibliothek genießen zu dürfen. Die Worte sind abgegriffen, aber die Erinnerungen sind noch frisch und eine Quelle anhaltender Freude.

Schließlich möchte ich Ingo Gildenhart und Andrew Zissos für ihre Hilfe bei der Erstellung des Registers und dem Korrekturlesen dieses Manuskripts danken. Ich habe ihren hohen Maßstäben und ihrer Bereitwilligkeit viel zu verdanken. 